\title{
Outcome of surgical untethering of tethered cord syndrome in adults due to spina bifida occulta
}

Dr. Bassam Mahmood Flamerz Arkawazi

Background: Children with spina bifida occulta require early surgery to prevent neurological deficits. The treatment of patients with a congenitally tethered cord who present in adulthood remains controversial.

Objective: The aim of this study is to describe the outcome obtained in 61 adult patients with congenital TCS and no prior surgical treatment who underwent surgical untethering.

Methods: This prospective study was conducted on 61 adult patients who underwent surgical untethering for spina bifida occulta at four neurosurgical centers in Baghdad / Iraq between March 2000 and January 2018. Patients who had undergone prior myelomeningocele repair or tethered cord release surgery were excluded.

The most common intraoperative findings were lipomyelomeningocele $(41 \%)$ and a tight terminal filum (36\%). The follow-up duration ranged from 10.8 to 149.5 months (mean 20.9 months). Of the 34 patients with back pain, status improved in $65 \%$, worsened in $3 \%$, remained unchanged in $18 \%$, and improved and later recurred in $15 \%$. Lower-extremity pain improved in 16 patients $(53 \%)$, remained unchanged in $23 \%$, improved and then recurred in $17 \%$, and worsened in $7 \%$. Lowerextremity weakness improved in $47 \%$, remained unchanged in $47 \%$, and improved and then recurred in 5\%. Finally, of the 17 patients with lower-extremity sensory changes, status improved in 35\%, remained unchanged in $35 \%$, and the information on five patients was unavailable. Surgical complications included three wound infections, one cerebrospinal fluid leak, and two pseudomeningoceles requiring surgical revision. One patient developed acute respiratory distress syndrome and sepsis postoperatively and died several days later.

Conclusions: Adult-age presentation of a congenital tethered cord is unusual. Despite a slight increase in postoperative neurological injury in adults, surgery has relatively low risk and offers good potential for neurological improvement or stabilization. As in children, we recommend early surgery in adults with this disorder. The decision to undertake surgery, however, should be modulated by other factors such as a patient's general medical condition and risk posed by anesthesia.

Key words: tethered cord syndrome - spina bifida occulta , congenital disease , Surgery

Corresponding address to:

Assistant Professor of Neurosurgery

Alkindy College of Medicine

University of Baghdad

Received at 7/1/2019

Accepted at 29/1/2019

\section{INTRODUCTION}

Occult spinal dysraphism refers to a spectrum of congenital anomalies that arise from the incomplete formation of posterior midline structures during embryogenesis. These disorders cause a multitude of neurological

deficits secondary to rostrocaudal traction on the spinal cord. Garceau ${ }^{(1)}$ first described the "filum terminale syndrome" in 1953 in three patients. Two decades later, in 1976, Hoffman and colleagues ${ }^{(2)}$ coined the term "tethered spinal cord" to describe the disorder. In 1981, Yamada et al. (3) adopted the term "tethered cord syndrome," (TCS) the terminology that is accepted today. The authors of several reports in the literature have established that appropriate early operative untethering of the spinal cord prevents the development, inhibits the progression, and even often reverses associated neurological symptoms. Symptoms related to a congenital tethered cord occur most commonly in childhood, but in many patients a diagnosis is never established until adulthood when the manifestation of symptoms encourages them to seek medical attention. Patients who never undergo treatment for TCS likely have an elevated risk of developing symptoms with advancing age. There are an increasing number of articles on the recognition of and surgical outcomes in adults with congenital TCS. In a literature review focusing on adult TCS by Lapsiwala and Iskandar, ${ }^{(4)}$ however, it was found that the authors of most large series reported "mixed" data in which patients with both spina bifida occulta and spina bifida aperta were included, or both adult and pediatric populations were reported. Of the numerous studies of adult TCS evaluated, they found only four in which the investigators reported "pure" adult 
spina bifida occulta information alone: Akay et al., ${ }^{(5)}$ Gupta

et al., ${ }^{(6)}$ Iskandar et al., ${ }^{(7)}$ and Filler et al. ${ }^{(8)}$ In the largest series to date of adult-onset spina bifida occulta, the authors evaluated 34 patients with no history of surgical intervention. ${ }^{(7)}$

The aim of this study is to describe the outcome obtained in 61 adult patients with congenital TCS and no prior surgical treatment who underwent surgical untethering.

\section{METHODS}

\section{Patient Population}

We reviewed the medical records of 61 adult patients with congenital TCS who underwent surgery between March 2000 and January 2018 at four different neurosurgical centers in Baghdad / Iraq. The study population consisted of patients with congenital, not acquired, spinal cord tethering, and therefore any patient presenting with post-myelomeningocele tethered cord was excluded. Only patients over 17 years of age were included. Presentations that may have been complicated by other pathological processes, such as Chiari malformation or spinal cord arteriovenous malformations were excluded. The age of the patients remaining in this study ranged from 18 to 62 years (mean 36 years). There were 16 male and 45 female (male/female ratio 1:2.8). The follow-up period ranged from 10.8 to 149.5 months, with a mean of 20.9 months.

\section{RESULTS}

\section{Preoperative Signs and Symptoms}

Preoperatively, 34 patients (56\%) suffered back pain; 48 patients (79\%) suffered lower-extremity dysfunction, including weakness, pain, numbness, and/or atrophy; 21 patients (34\%) complained of bowel and/or bladder dysfunction: and two patients (3\%) experienced sexual dysfunction, including retrograde ejaculation and dyspareunia. In one patient (2\%) a tethered cord that had never caused neurological deficits or symptoms was diagnosed during evaluation for a hairy patch found in the lumbar region. One patient was noted to have bilateral and four patients unilateral lower-extremity atrophy at the time that the diagnosis of tethered cord was made.

\section{Surgical Treatment}

In all cases the surgery consisted of tethered cord release (TCR) in which the tethering lesion was excised when appropriate. The spinal defects repaired included lipomyelomeningoceles (25 patients), tight terminal fila

(22 patients), split cord malformation (SCM) (15 patients), syringomyelia (seven patients), dermoid cysts (two patients), and a meningocele (one patient) (Fig. 1). In 12 patients $(20 \%)$ we documented more than one anomaly. Seven patients had scoliosis and one patient had a lumbar spondylosis that had previously been corrected.

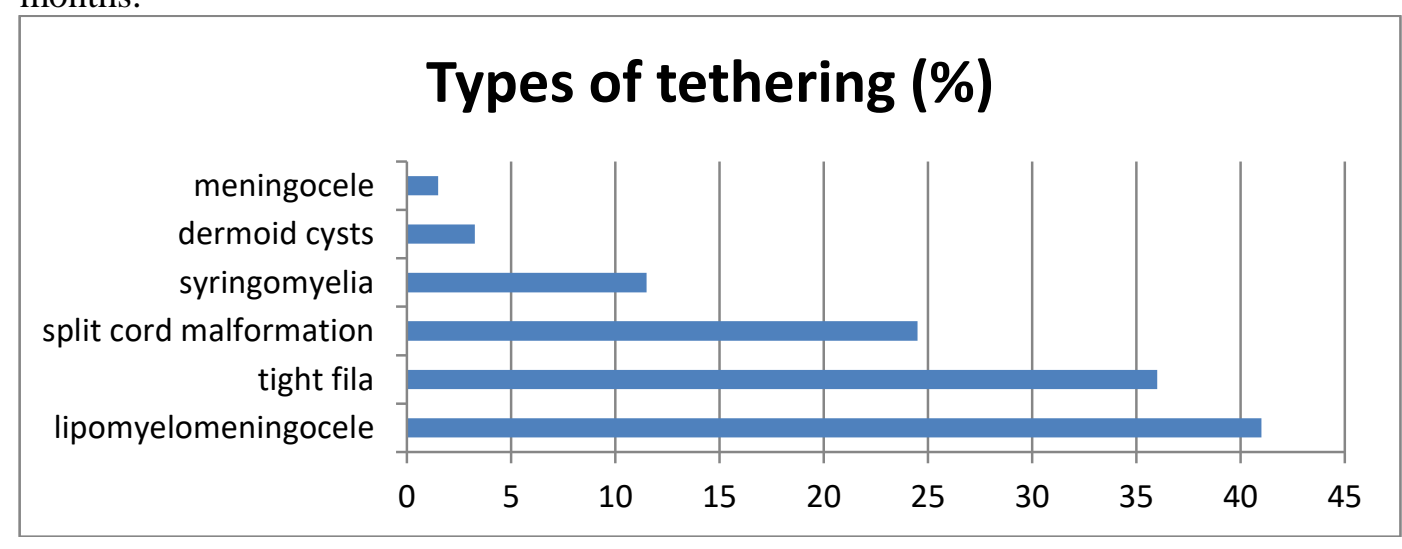

FIG. 1. Bar graph demonstrating the percentages of adult patients with various spinal anomalies noted intraoperatively.

The duraplasty technique and type of graft material differed among surgeons and changed over the years. The thecal sac was reconstructed in 23 patients. Synthetic dura mater was used 
in 12 patients, and fascia lata in eight patients. In three cases the information on graft type was not available.

The most common surgical outcomes are summarized in table 1 .

Table 1. Surgical outcomes in adult patients after tethered cord release stratified by presenting signs and symptoms

\begin{tabular}{|l|l|l|l|l|l|l|}
\hline & \multicolumn{7}{|c|}{ Signs and symptoms \% } \\
\hline Signs and symptoms & improved & stable & worse & Recurrence & Unknown & total \\
\hline Back pain & $22(64.7)$ & $6(17.6)$ & 1 & $5(14.7)$ & $0(0)$ & 34 \\
& & & $(2.9)$ & & & \\
\hline Lower extremity dysfunction & $22(45.8)$ & 17 & 2 & $7(14.6)$ & $0(0)$ & 48 \\
Pain & $16(53.3)$ & $(35.4)$ & $(4.2)$ & $6(16.7)$ & $0(0)$ & 30 \\
Weakness & $9(47.4)$ & $7(23.3)$ & 2 & $1(5.3)$ & $0(0)$ & 19 \\
numbness & $6(35.3)$ & $9(47.4)$ & $(6.7)$ & $0(0)$ & $5(29.4)$ & 17 \\
& & $6(35.3)$ & $0(0)$ & & & \\
& & & $0(0)$ & & & \\
\hline Bowel/bladder dysfunction & $13(61.9)$ & $6(28.6)$ & 1 & $1(4.8)$ & $0(0)$ & 21 \\
& & & $(4.8)$ & & & $0(0)$ \\
\hline Sexual dysfunction & $1(50)$ & $1(50)$ & $0(0)$ & $0(0)$ & 2 \\
\hline
\end{tabular}

Of the 34 patients with back pain, 22 $(65 \%)$ improved, six $(18 \%)$ remained unchanged, one (3\%) worsened, and five (15\%) experienced an initial improvement but then had a recurrence of the symptom. Of the 48 patients with lower-extremity dysfunction, the status improved in 22 (46\%), remained unchanged in $17(35 \%)$, worsened in two (4\%), and improved but then recurred in seven (15\%). Analyzing each lower-extremity symptom individually, 30 patients complained of pain, 19 suffered weakness, and 17 experienced numbness. Symptoms in the 16 patients $(53 \%)$ with lower-extremity pain improved, remained unchanged in seven (23\%), worsened in two (7\%), and improved but recurred in five $(17 \%)$. Lower-extremity weakness improved in nine patients $(47 \%)$, remained unchanged in nine $(47 \%)$, and improved but recurred in one (5\%). Finally, of those patients with lower-extremity numbness, the status improved in six (35\%), remained unchanged in six (35\%), and the information for five (29\%) was not available.

Within the group of 21 patients with bowel and/or bladder dysfunction, 13 (62\%) improved, six (29\%) remained unchanged, one (5\%) worsened, and one (5\%) experienced initial improvement but a late recurrence. The one patient with upper-extremity pain and numbness experienced an improvement in symptoms after surgery. Finally, of the two patients with sexual dysfunction, the symptom in the patient with dyspareunia improved whereas that in the patient with retrograde ejaculation remained unchanged. In three patients who experienced initial symptomatic improvement after undergoing tethered cord release, two suffered symptom recurrence after a motor vehicle accident, and one suffered a recurrence after falling down.

Complications of the surgical treatment included postoperative wound infection in three patients, CSF leakage in one, and development of a pseudomeningocele in two patients (total of six patients, or $10 \%$ of the overall population). Two of the wound infections were treated with antibiotic alone and one was treated with a wound revision. All three patients in whom a pseudomeningocele or CSF leak developed required wound revision. After surgery, one patient had difficulty micturating and required selfcatheterization. This particular patient had a highly calcified dermoid tumor enwrapping the cauda equina. One patient developed acute respiratory distress syndrome and sepsis postoperatively and died several days after surgery. There were no cases of meningitis. 


\section{DISCUSSION}

Adult Compared With Pediatric
Presentation

Tethered cord syndrome involves a progression of neurological

dysfunction secondary to spinal cord traction. It is well established that early surgical intervention of a congenitally tethered spinal cord in children affords benefit through prevention of neurological deterioration. A surgeon's experience and the technical advances in the field of pediatric neurosurgery have reduced the mortality and morbidity rates associated with such repairs. Although symptoms develop most commonly in childhood, they can occasionally occur in adulthood. The number of adults in whom congenital TCS is diagnosed continues to grow as a result of better imaging and recognition of this syndrome. Pediatric TCS has been well studied in the literature, but much of the information regarding the adult population is still being defined. In this study, we have described the experience of surgeons at four neurosurgical centers who, between March 2000 and January 2018, performed surgical untethering operations in 61 adult patients with congenital adult TCS. Any patient who underwent previous spinal surgery, whether for

repair of a myelomeningocele or spina bifida occulta, was excluded from this study.

The spinal defects observed in adult patients are similar to those seen in the childhood syndrome and include a lipomyelomeningocele or spinal cord lipoma, split cord malformation, thick/fatty terminal filum, meningocele, terminal syringohydromyelia, dermoid cyst, ventral bands, neurenteric cyst, atretic meningocele, and dermal sinus tract. ${ }^{(7,9)}$ These lesions may be found alone or in various combinations. Spinal cord anomalies are often associated with cutaneous changes on the midline of the back-namely, a dermal sinus, hypertrichosis, subcutaneous lipoma, atretic meningocele, caudal appendage, or hemangioma. In their original series of 200 adults and children with spina bifida occulta, James and Lassman ${ }^{(10)}$ found that as many as 32 had no cutaneous stigmata. Similarities and differences exist between the adult and pediatric populations, and in many regards certain disparities between the two may be attributed to the younger child's inability to communicate symptoms such as pain, sensory changes, urinary urgency, incomplete voiding, or incontinence. $^{(9)}$ Unlike their pediatric counterparts, adults also experience a more gradual and insidious onset of symptoms, typically over the course of several months to years. For this reason, the true incidence of adult congenital TCS remains unknown - many of these patients are either overlooked or never seek medical attention. ${ }^{(11)}$ The severity of TCSinduced deficits can often be underrated or even misdiagnosed for years. One illustration of this phenomenon was the case of a 43-year-old woman with a several- year history of perianal numbness, gluteal radicular pain, low-back pain, bowel incontinence, and a lifelong history of bladder incontinence for which she had undergone reflux surgery. She was found to have a lipomyelomeningocele and underwent surgical intervention. Postoperatively her symptoms improved. Because degenerative disc disease and low-back pain are very common problems in adults, the diagnosis of a symptomatic tethered cord is often misattributed to other entities and thereby delayed. ${ }^{(12)}$ In their analysis of 57 adult patients with TCS due to various causes, van Leeuwen et al. ${ }^{(13)}$ reported a mean delay in diagnosis of 7 years and a mean delay in surgery after diagnosis of up to 4 more years. The diagnosis with new symptom onset has even been reported in the elderly; for instance, Sostrin and coworkers ${ }^{(14)}$ described a previously asymptomatic 75year-old man with a 6-month history of ambulatory difficulty and lower-extremity weakness; he was found to have spinal stenosis and a TCS secondary to a lipomyelomeningocele.

Patients with TCS present with various forms of neurological deficits-from those involving a combination of upper and lower motor neurons to variable sensory involvement. The range of clinical symptoms can be incapacitating and 
include back pain and sensory deficits (often radiating to the buttocks and legs), muscle weakness, bowel dysfunction, bladder dysfunction, and skeletal and foot deformities. ${ }^{(5,6,7,8)}$ Bowel dysfunction can include incontinence or frequency. In their evaluation of 15 adults with TCS of varying origins, Kondo and associates ${ }^{(15)}$ reported urological symptoms to include impaired detrusor contractility, residual urine ( $>50 \mathrm{ml}$ ), incontinence (urge, stress, or bed wetting), sensory urgency, vesicoureteral reflux, urinary tract infections, impotence, and moderate renal impairment. The most common clinical presentations in our series were back pain and lower-extremity pain, with one third of the population presenting with bladder dysfunction and one third with neurological changes in the lower extremities. Although pediatric patients present more often with bladder dysfunction, adults tend to present mostly with pain. ${ }^{(9,16)}$ Typically, this consists of a very characteristic constellation of symptoms: nondermatomal pattern, shock like sensations, and a burning quality. ${ }^{(17)}$ Leg and foot anomalies occur as a result of an imbalance between muscles, bones, and joints during growth, typically resulting in a pes cavus deformity. ${ }^{(2)}$ These congenital anomalies develop in some children with TCS and persist into adulthood but are not believed to occur in adults de novo.

\section{Surgical Management}

There is much debate over the surgical management of congenital TCS presenting in adulthood. Whereas some authors advocate prophylactic surgery, others suggest that surgery should be performed only when symptom progression develops. In adults, however, the diagnosis is typically established only after the individual becomes symptomatic or experiences a progression of already existing symptoms. This effectively reduces the number of patients in whom the diagnosis is made incidentally (in our series, only one patient was asymptomatic).

Many surgeons believe that adults and children should be considered separately because, although they have a similar underlying pathological condition, their mechanism of symptom occurrence may be different. A debatable hypothesis is that children may present with progressive neurological deficits as a result of unequal growth between the spinal cord and vertebral column that causes increasing caudal spinal cord traction; adults, however, do not experience this because they have completed their growth curve. Instead, repetitive extension and flexion of the spine may cause unremitting traction on a tethered spinal cord. ${ }^{(18)}$ Over time, this continuous traction produces local spinal cord ischemia and neuronal damage with worsening vascular and metabolicdysfunction. ${ }^{(3,19)}$ Whereas some adults present with progressive symptoms, others present with much more severe and disabling neurological symptoms due to an acute, aggravating event. The age at which a symptom manifests may in fact be related to the degree of cord traction. Pang and Wilberger ${ }^{(9)}$ divided their patients into three pathophysiological groups: 1) momentary stretching of a tight conus medullaris, typically due to extreme hip flexion during situations such as childbirth, sexual intercourse, or a motor vehicle accident; 2) a narrow spinal canal with crowding of intraspinal contents and a tight conus medullaris, such as in the presence of an associated disc herniation or spinal stenosis; and 3) a direct blow to the back or a fall onto the buttocks. A tight conus medullaris is typically characterized by a low-lying conus medullaris or the presence of a thick $(>2-\mathrm{mm})$ and fatty terminal filum. ${ }^{(4)}$ Prolonged forward bending or sitting can also exacerbate symptoms. With the spinal cord already in traction, these and similar events cause a sudden pull on the spinal cord, inducing further spinal cord injury. At times multiple factors may coexist. In patients who have undergone surgical repair, it has been postulated that neurological progression is a result of evolving degenerative spinal disease, perhaps explaining why pain is the most commonly reported symptom in adults. ${ }^{(8)}$ The goal of surgical intervention is twofold: 1) to prevent further neurological exacerbation, and 2) to improve the patient's symptoms, especially pain, if possible. The surgical outcomes for adults 
who have undergone TCR are encouraging. In our series, of the 106 total symptoms with which patients presented, there was an overall improvement rate of $56 \%$, a stabilization rate of $28 \%$, and a worsening rate of only $4 \%$. Approximately $12 \%$ of the symptoms improved but recurred in a delayed fashion. There were no reported intraoperative complications, although four patients (7\%) were taken back to the operating room to undergo wound revisions due to an infection, CSF leakage, or pseudomeningocele. This complication rate is similar to that reported by others $(11-17 \%) .^{(7,20)}$ The reported mortality rate for TCR is also low. There was one patient in

our study who died several days postoperatively of acute respiratory distress syndrome and sepsis. The use of a graft or the type of graft for dural repair did not appear to correlate with postoperative morbidity or death.

Symptoms that improved the most after surgical correction were back pain $(65 \%)$ and bowel/bladder dysfunction (62\%). Such a high rate of postoperative improvement in bowel/bladder function is unusual, however, because some authors believe that bowel and bladder difficulties are the least likely of all symptoms to respond to surgery. ${ }^{(21)}$

\section{CONCLUSION}

Although a prospective study of 61 patients does not provide management standards, a study of this size supplies useful information on demographic features, clinical presentation, and surgical complication rates. Conversely, indications for and long-term results of surgery are best obtained from randomized controlled studies. Such studies are impracticable because of the paucity of adults with TCS, as well as ethical concerns regarding the idea of randomizing symptomatic individuals to conservative treatment. Nevertheless, whereas we in no way propose that all adult patients with a congenital tethered cord should undergo surgery, we do argue, based on the findings, that some of these adults will benefit from such treatment. It has indeed become evident over the past few years that individuals with spina bifida occulta who have achieved full growth may suffer neurological deterioration after sudden flexion (for example, a motor vehicle accident or lithotomy position). In addition, it is notable that the vast majority of patients in this series were symptomatic or already had neurological deficits, which argues against a benign natural history for this disease process. Because it is difficult, if not impossible, to distinguish between patients in whom symptoms will deteriorate and those in whom they will not, a rational approach would be to treat all with neuroimaging evidence of cord tethering, as long as the medical and anesthetic risks are low.

\section{REFRENCES}

1. Garceau GJ: The filum terminale syndrome (the cord-traction syndrome). J Bone Joint Surg Am 35:711-716, 1953

2. Hoffman HJ, Hendrick EB, Humphreys RP: The tethered spinal cord: its protean manifestations, diagnosis and surgical correction. Childs Brain 2:145-155, 1976

3. Yamada S, Zinke DE, Sanders D: Pathophysiology of "tethered cord syndrome." J Neurosurg 54:494-503, 1981

4. Lapsiwala SB, Iskandar BJ: The tethered cord syndrome in adults with spina bifida occulta. Neurol Res 26:735-740, 2014

5. Akay KM, Ersahin Y, Cakir Y: Tethered cord syndrome in adults. Acta Neurochir (Wien) 142:1111-1115, 2016

6. Gupta SK, Khosla VK, Sharma BS, Mathuriya SN, Pathak A, Tewari MK: Tethered cord syndrome in adults. Surg Neurol 52:362-369, 1999

7. Iskandar BJ, Fulmer BB, Hadley $\mathrm{MN}$, Oakes WJ: Congenital tethered spinal cord syndrome in adults. J Neurosurg 88: 958-961, 1998

8. Filler AG, Britton JA, Uttley D, Marsh HT: Adult postrepair myelomeningocoele and tethered cord syndrome: good surgical outcome after abrupt neurological decline. Br J Neurosurg 9: 659-666, 1995

9. Pang D, Wilberger JE Jr: Tethered cord syndrome in adults. J Neurosurg 57:32-47, 1982

10. James CCM, Lassman LP: Spina Bifida Occulta: Orthopaedic, Radiological and Neurosurgical Aspects. London: Academic Press, 1981

11. English WJ, Maltby GL: Diastematomyelia in adults. J Neurosurg 27:260-264, 1967 
12. Loeser JD, Lewin RJ: Lumbosacral lipoma in the adult: case report. J Neurosurg 29:405409, 1968

13. van Leeuwen R, Notermans NC, Vandertop WP: Surgery in adults with tethered cord syndrome: outcome study with independent clinical review. J Neurosurg 94 (2 Suppl):205-209, 2017

14. Sostrin RD, Thompson JR, Rouhe SA, Hasso AN: Occult spinal dysraphism in the geriatric patient. Radiology 125:165-169, 1977

15. Kondo A, Kato K, Kanai S, Sakakibara T: Bladder dysfunction secondary to tethered cord syndrome in adults: is it curable? J Urol 135:313-316, 1986

16. Yamada S, Knierim D, Yonekura M, Schultz R, Maeda G: Tethered cord syndrome. J Am Paraplegia Soc 6:58-61, 1983
17. Huttmann S, Krauss J, Collmann H, Sorensen N, Roosen K: Surgical management of tethered spinal cord in adults: report of 54 cases. J Neurosurg 95 (2 Suppl):173-178, 2015

18. Garcia FA, Kranzler LI, Siquiera EB, Weinberg PE, Kranzler KJ: Diastematomyelia in an adult. Surg Neurol 14:93-94, 1980

19. Yamada S, Iacono RP, Andrade T, Mandybur G, Yamada BS: Pathophysiology of tethered cord syndrome. Neurosurg Clin N Am 6:311-323, 1995

20. Klekamp J, Raimondi AJ, Samii M: Occult dysraphism in adulthood:

clinical course and management. Childs Nerv Syst 10: 312-320, 1994

21. Salvati M, Ramundo EO, Artico M, Martini S, Caruso R, Fortuna A: The tethered cord syndrome in the adult. Report of three cases and review of the literature. Zentralbl Neurochir 51:91-93, 1990 